\title{
Strain-Rate Effect in Rapid Triaxial LoAding OF Concrete
}

\author{
By Zdenĕk P. Bažant,' F. ASCE and Byung H. Oh'
}

\begin{abstract}
Assmacr: Presented is a generalization of time-independent nonlinear triaxial constitutive relations for concrete to model short-time viscuelastic effects. The effect of strain-rate magnitude upon the initial elastic modulus. the peak stress, and the sharpness of the stress peak is taken into account. To obtain a model that also exhibits short-time rapid creep and stress relaxation, the incrementally linear constitutive law fully characterized by tangential moduli is replaced by a nonlinear first-order differential equation in stress, strain, stress rate and strain rate. This requires introduction of the (apparent) instantaneous modulus, the relaxation time and retardation time. The irreversibility at unloading, caused by the short-time viscoelastic effects, is also analyzed. The model allows more realistic dynamic finite element analysis of concrete structures.
\end{abstract}

\section{INTAODUCTION}

In dynamic finite element analysis of concrete structures subjected to blast, impact, earthquake, or nuclear accident loading, the stiffness of material may significantly depend on the strain rate. This effect has been studied extensively $(1,2,20,21,23-25,27,28-39,41-43,46-53,55-64)$. However, most studies have been limited to uniaxial dynamic behavior, and a realistic triaxial constitutive model is unavailable at present. We set it, therefore, as our objective to develop such a model by generalizing an existing rate-independent (or time-independent) nonlinear triaxial constitutive model for concrete, such as the plastic-fracturing theory $(7,12)$ or the endochronic theory $(6,9,10,18,19)$.

In diverse practical applications, the strain rates can be of rather different orders of magnitudes, as, e.g., for impact compared with earthquake. To cover all these applications, the usual approach of viscoelasticity would call for a rheologic model (such as the Maxwell or Kelvin chain) with a very broad relaxation or retardation spectrum. This would, however, force us to consider for each material point a number of internal variables (e.g., the partial stresses of Maxwell chain or the partial strains of Kelvin chain). Such an approach, which was actually taken for the endochronic theory in Ref. 8, is, however, rather inconven-

'Prof. of Civ. Engrg. and Dir., Center for Concrete and Geomaterials, Northwestern Univ., Evanston, Ill. 60201.

${ }^{2}$ Grad. Research Asst., Northwestern Univ., Evanston, III. 60201.

Note--Discussion open until March 1, 1983. To extend the closing date one month, a written request must be filed with the Manager of Technical and Professional Publications, ASCE. Manuscript was submitted for review for possible publication on October 1, 1980. This paper is part of the Journal of the Engineering Mechanics Division, Proceedings of the American Society of Civil Engineers, CASCE, Vol. 108, No. EM5, October, 1982. ISSN 0044-7951/82/0005-0764/\$01.00. 
ient in case of a large finite element program, since the number of variables increases many times. Therefore, we will try to develop here a constitutive model that is applicable for many different orders of strain-rate magnitude, yet does not employ any internal variables.

\section{Aojusting Thanxial Constitutive Relation to Umiaxial Data}

The parameters in the recently developed endochronic $(6,7,9,10,18,19)$ and plastic-fracturing (12) constitutive equations for concrete were expressed as functions of a single characteristic of the material, the standard cylinder strength, $f_{c}^{\prime}$. In general, these constitutive equations may be written as

$\Phi_{i j}\left[\boldsymbol{\sigma}(z), \epsilon(z) ; f_{c}^{\prime}\right]=0$

in which $\sigma=$ stress tensor; $\epsilon=$ strain tensor (only small strains are considered here); $\Phi_{i j}=$ a tensor-valued functional of the histories $\sigma(z)$ and $\epsilon(z)$ from the moment of first loading to the current state; lower case latin subscripts refer to cartesian coordinates $x_{i}(i=1,2,3)$; and $z=$ the history parameter. For time and rate-independent behavior, $z$, for example, may be considered as the length of the path traced in the strain space. The endochronic theory $(6,9,10,18,19)$ and the plastic-fracturing theory (12) are two examples which are covered by Eq. 1. In particular, for the plastic-fracturing theory, the constitutive relation can be reduced to the incrementally linear history-independent form (12):

$d \sigma_{i j}=C_{i j k m}\left(\sigma, \epsilon ; \quad f_{c}^{\prime}\right) d \epsilon_{k m}$

in which $\sigma_{i j}$ and $\epsilon_{\mathrm{km}}=$ the components of tensors $\sigma$ and $\epsilon$; repetition of subscripts implies summation; and $C_{i j k m}=$ the fourth-rank tensor of tangential moduli which differ for loading and unloading, depend on the current values of $\sigma$ and $\epsilon$, and may be determined on the basis of Eqs. 21, 22, and 30-34 of Ref. 12. Note that the endochronic theory can also be linearized (6) to reduce it to Eq. 2, but only for a limited range of loading directions in the strain space.

The shape of the uniaxial stress-strain curve of concrete depends on the strength, $f_{c}^{\prime}$. Generally, for a lower strength, the inelastic strains at the same stress-strength ratio are larger, and the peak portion of the curve is flatter and more extended. For a higher strength, the peak becomes sharper. These features may be characterized by the parameter (Fig. 1(a))

$p=\frac{E \epsilon_{p}}{\sigma_{p}}$

in which $E=$ initial elastic modulus (slope of the initial tangent); $\sigma_{p}=$ peak stress (which coincides with $f_{c}^{\prime}$ in case of a static test); and $\epsilon_{p}=$ strain at peak stress. Parameter $p$ represents the ratio of strain at peak stress to the elastic strain, $\sigma_{p} / E$, for this stress and characterizes the offset of the stress peak point from the initial tangent. The constitutive relation in Eq. 1 or 2 yields a uniaxial stressstrain curve which is characterized by three basic parameters: peak stress, $\sigma_{p}$ $=f_{c}^{\prime}$, initial elastic modulus, $E$, and parameter $p$, i.e. 

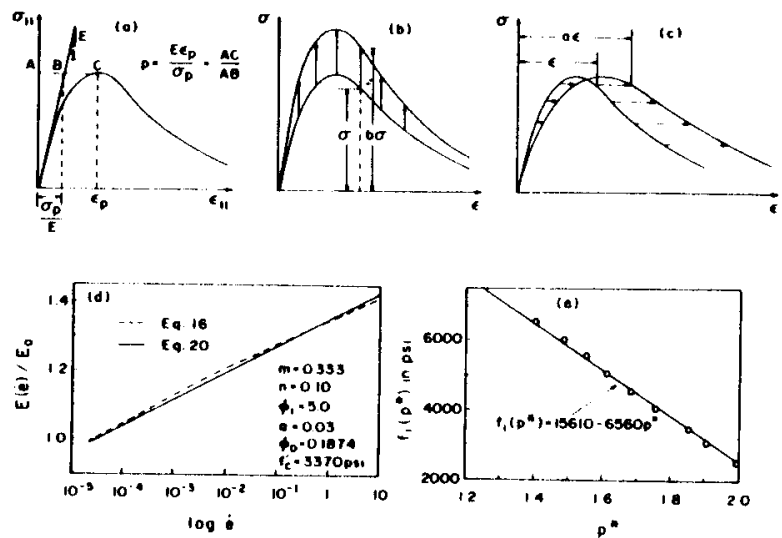

100

FIG. 1.-Charecteristics of: (a) Coefficient $p$; (b) Coefficient $b$; (c) Coefficient $a$; (d) Function $E(e)$; (o) Relation between $f_{1}\left(p^{*}\right)$ and $p^{*}\left(1 \mathrm{psi}=6,895 \mathrm{~N} / \mathrm{m}^{2}\right)$

We will now study the following problem: How should we transform Eq. 1 or Eq. 2 to yield certain required values

$E^{*}, \sigma_{p}^{*}, p^{*}$

of these three parameters? We will need this to determine the changes in these parameters caused by a change in the strain rate.

The existing endochronic and plastic-fracturing formulations for concrete do not involve parameter $p$ and yield no closed-form expression for the uniaxial stress-strain curve. Thus, to obtain the dependence of parameter $p$ on $f_{c}^{\prime}$, we may consider various values of $f_{c}^{\prime}$ and for each we may run a computer program (12) to obtain the uniaxial stress-strain curve, from which we evaluate $p$. Then we may plot all the points $\left(f_{c}^{\prime}, p\right)$ (Fig. $1(e)$ ), and by fitting an empirical expression to the points (see Eq. 19 in the sequel), we may obtain the function

$f_{c}^{\prime}=f_{1}(p)$

By a similar process or by using the expression for $E$ given in Eq. 20 of Ref. 9 or Eq. 34 of Ref. 12, we may obtain the relation $E=E\left(f_{c}^{\prime}\right)$ from which, by using Eq. 6, we get the function (see Eq. 20 in the sequel):

$E=f_{2}(p)$

Consider now that the value $p^{*}$, which characterizes the desired shape of the uniaxial stress-strain curve and generally differs from the value $p$ for the static uniaxial test, is given. Substituting Eqs. 6 and 7 into Eqs. 1 and 2, we have

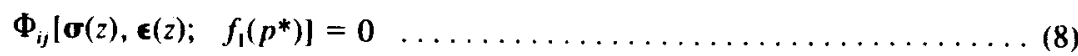

and $d \sigma_{i j}=C_{i j k m}\left[\sigma, \epsilon ; f_{1}\left(p^{*}\right)\right] d \epsilon_{k m}$

These constitutive relations will yield the peak stress, $f_{1}\left(p^{*}\right)$, and initial tangent modulus, $f_{2}\left(p^{*}\right)$.

We will now seek a transformation that preserves the value $p^{*}$ but changes the peak stress and the initial tangent modulus. This may be achieved by affinity transformations (Fig. $1(b, c)$ ), in which the strain values are multiplied by constant $1 / a$, and the stress values by constant $1 / b$, i.e., $\epsilon$ and $\sigma$ are replaced by $a \in$ and $b$ :

$\Phi_{i j}\left[b \sigma(z), a \in(z) ; \quad f_{1}\left(p^{*}\right)\right]=0$

and $b d \sigma_{i j}=C_{i j k m}$

$$
\left.a \in ; \quad f_{1}\left(p^{*}\right)\right] a d \epsilon_{k m}
$$

Note that parameter $p$ is independent of such transformations, while the peak stress, $f_{1}\left(p^{*}\right)$, and the initial tangent modulus, $f_{2}\left(p^{*}\right)$, get transformed to the values

$\sigma_{p}^{*}=\frac{1}{b} f_{1}\left(p^{*}\right) ; \quad E^{*}=\frac{a}{b} f_{2}\left(p^{*}\right)$

in which, for the static test, $\sigma_{p}=f_{c}^{\prime}=f_{1}(p)$. From these relations, we determine the tranformation coefficients, $a$ and $b$, needed to get the desired values, $\sigma_{p}^{*}$ and $E^{*}$, at prescribed peak stress offset, $p^{*}$ :

$b=\frac{f_{1}\left(p^{*}\right)}{\sigma_{p}^{*}} ; \quad a=\frac{E^{*}}{f_{2}\left(p^{*}\right)} b$

\section{Truaxial Stress-Strain Relation with Stanin-Rate Effect}

When measurements are conducted at various constant strain rates, the observed stress-strain relations are similar in shape and the effect of the strain rate is manifested mainly in the value of the peak stress (strength), $\sigma_{p}^{*}$, the initial elastic modulus, and the sharpness of the peak, as characterized by parameter $p^{*}(1,2,23,27,28,30-39,41,42,49,51,52,57-64)$. Concerning the dependence of the triaxial aspects of the deformation (e.g., inelastic dilatancy, shear compaction, lateral strain ratio) on the strain rate, no experimental information seems to exist and it is reasonable to assume at present that these aspects are approximately independent of the strain rate. We will, therefore, assume that the effect of various constant strain rates in triaxial behavior is adequately described by adjusting the values of $\sigma_{p}^{*}, p^{*}$, and $E^{*}$. This can be done on the basis of uniaxial tests alone.

In presence of viscoelastic effects, the meaning of the initial elastic modulus requires closer examination. At first, we might be tempted to interpret $E$ (or $E^{*}$ ) as the instantaneous modulus, which corresponds to an instantaneous load application. Such a definition of $E$ would, however, cause unnecessary complications. It would force us to calculate the viscoelastic effects for times that are orders of magnitude shorter than the time to reach the peak stress. This is because concrete does not have a narrow relaxation spectrum and exhibits viscoelastic response for load durations much less than $1 / 10$ of the time to reach the peak stress. However, when we consider a stress-strain curve with strain $\epsilon_{p}$ at peak stress, we do not need to describe the viscoelastic effects accurately for very small strains, e.g., for $0.001 \epsilon_{p}$. It is sufficient to describe them well for strains larger than about $0.05 \epsilon_{p}$. Therefore, we propose to define approximately the initial tangent modulus as the secant modulus for strain $0.05 \epsilon_{p}$. 
If we consider the strains less than $0.05 \epsilon_{p}$, we may treat concrete as linearly viscoelastic. It is then characterized by the compliance function, $J\left(t, t^{\prime}\right)$, (also called the creep function), which is defined as the strain at time $t$ produced by a constant unit stress of duration $t-t^{\prime}(3,4,5,8,11,13-17)$. The behavior of concrete is rather well represented by

$J\left(t, t^{\prime}\right)=\frac{1}{E_{0}}\left[1+\phi_{0}\left(t-t^{\prime}\right)^{n}\right] ; \quad \phi_{0}=\phi_{1}\left(t^{-m}+\alpha\right)$

called the double power law $(5,16,17)$, in which $t^{\prime}=$ age of concrete at time of loading, and $E_{0}=$ asymptotic modulus (limit value for instantaneous loading). The values of material parameters $\phi_{1}, n, m, \alpha$, and $E_{0}$ are indicated in Ref. 17; typical values are $m \simeq 1 / 3, n \simeq 0.135, \alpha \approx 0.05, \phi_{1}=2-8$ (approx), if times are given in days; $E_{0}=1.5 E_{28} ; E_{28}=$ standard 28-day elastic modulus. Although Eq. 14 was determined primarily from long-time creep data, it was shown that it gives roughly correct values also for rapid creep (load durations from 1 sec up) and even for the dynamic modulus $(16,17)$. This permits us to use Eq. 14 for our purpose, and an independent determination of $E$ from Eqs. 7, 17, and 20 will later confirm that the results are correct.

Since the aging of concrete during rapid loading is negligible, the response to a variable stress may be quite well approximated by the effective modulus, defined as $E_{\text {eff }}=1 / J\left(t, t^{\prime}\right)(6,7,13)$. Therefore, we may take the initial tangent modulus, $E^{*}$, as the effective modulus for load duration equal to the time to reach strain $0.05 \epsilon_{p}$. For a uniaxial test, this time is $t-t^{\prime}=0.05 \epsilon_{p} / \dot{e}$, in which $\dot{e}$ is the absolute value of the uniaxial strain rate. For simplicity, we may assume here the value of $\epsilon_{p}$ for the static test, for which $\epsilon_{p} \approx 0.002$ is an adequate approximation for most concretes. Substituting for $t-t^{\prime}$ in Eq. 14, we get $t$ $-t^{\prime} \approx\left(10^{4} \dot{e}\right)^{-1}$ (days), and converting this from days to seconds, we obtain

$\frac{1}{E^{*}} \simeq J\left(t^{\prime}+\frac{10}{\dot{e}}, t^{\prime}\right)=\frac{1}{E_{0}}\left[1+\phi_{0}(0.1 \dot{e})^{-n}\right] \ldots \ldots \ldots \ldots \ldots \ldots \ldots \ldots$

For the static uniaxial test, which is normally conducted roughly at the rate $\dot{e}$ $\simeq 3.3 \times 10^{-5} / \mathrm{sec}(1,27)$, we get $\left.1 / E \simeq 11+\left(3.3 \times 10^{-6}\right)^{-n} \phi_{0}\right] / E_{0}$, and so, the relative change of initial tangent modulus $E^{*}$ for strain rate $\dot{\epsilon}$, compared with the static test, is

$\frac{E^{*}}{E}=\frac{1+\left(3.3 \times 10^{-6}\right)^{-n} \phi_{0}}{1+\left(0.1 e^{-n} \phi_{0}\right.}$

in which the strain rate must be given as the strain per second.

The following formulas have been established by fitting the available test data $(1,2,23,27,35,41,42,51,63,64)$ :

$p^{*}=2.09-0.00015 \sigma_{p 0}+f_{3}(\dot{e}) ; \quad f_{3}(\dot{e})=\frac{1-e^{1 / 8}}{1.84+3.2 \dot{e}^{-1 / 8}} \ldots \ldots \ldots \ldots$

$\sigma_{p}^{*}=\sigma_{p 0}\left[1.4-1.5 f_{3}(\dot{e})\right]$

in which $\sigma_{p 0}=$ the uniaxial strength in a normal static test, given in pounds per square inch $\left(1 \mathrm{psi}=6,895 \mathrm{~N} / \mathrm{m}^{2}\right)$. These expressions have been verified for $\dot{e}$ ranging from $10^{-5} / \mathrm{sec}$ to $1 / \mathrm{sec}$, but they give reasonable values even for the limits $\dot{e} \rightarrow 0$ and $\dot{e} \rightarrow \infty$ (e.g., Eq. 18 gives $\sigma_{p}^{*}=0.8 \sigma_{p u}$ for a constant $\dot{e}$ test that reaches failure strain within a few days, and $0.73 \sigma_{p 0}$ for $\dot{e} \rightarrow 0$ ). Within the range $10^{-5} / \mathrm{sec}$ to $1 / \mathrm{sec}$, nearly exactly the same values could be obtained with the simpler expression, $f_{3}(\dot{e})=-0.06 \log \dot{e}$, but the limits for $\dot{e} \rightarrow 0$ and $\dot{e} \rightarrow \infty$ would then be unreasonable, yielding $\pm \infty$ for $p^{*}$ and $\sigma_{p}^{*}$. The limit for $\dot{e} \rightarrow 0$ is not unimportant for a step-by-step computer program which uses the current value of $\dot{e}$; this is because $\dot{e} \approx 0$ can be obtained in some time step even for dynamic loading (at extreme displacements of oscillatory motion).

By making computer runs of the uniaxial stress-strain curves for various strengths, $\sigma_{p}\left(=f_{c}^{\prime}\right)$, and using the plastic-fracturing theory $(12)$, the following empirical expressions have been obtained for the functions in Eqs. 6 and 7 (see Fig. 1(e)):

$f_{1}\left(p^{*}\right)=\left(15,610-6,560 p^{*}\right)$

$f_{2}\left(p^{*}\right)=\left[0.9+0.00006 f_{1}\left(p^{*}\right)\right] 57,000 \sqrt{f_{1}\left(p^{*}\right)}$

(These expressions could also be used for other constitutive models, e.g., the endochronic theory.)

Since $E^{*}=f_{2}\left(p^{*}\right)(\mathrm{Eq} .7)$, we may obtain the dependence of elastic modulus $E$ upon $\dot{e}$ by substituting Eq. 17 into Eq. 20. However, this same dependence alternatively may also be obtained from Eq. 16 based on the creep law (Eq. 14). These two independent alternatives give almost the same results for $E^{*}$, as demonstrated in Fig. $l(d)$. This agreement strengthens the argument for our formulation.

One might now wonder why the relation of $E$ to $\dot{e}$ has not been determined directly from the test data on which Eq. 17 is based. The test data, however, do not indicate $E^{*}$ with sufficient accuracy because the strain, $0.05 \epsilon_{p}$, used to define $E^{*}$ is too small, causing too much scatter in the slope of the secant at $0.05 \epsilon_{p}$. One needs a formula for the entire curve in order to reduce the scatter of the initial secant slope.

An increase in $\dot{e}$ is known also to cause an increase in energy absorption (2), which is characterized by the area under the complete stress-strain curve. In our formulation, this effect is obtained as a consequence of the increase of peak stress. However, since a decrease in $p^{*}$ causes a decrease in the area under the curve, our theory predicts the area to increase in a smaller ratio than does the peak stress. Available dynamic test data on energy absorption are insufficient to confirm it at present; this is because, in such tests (2), the complete stressstrain curve has not been observed and the location of the terminal point on the curve apparently varied as a function of the test method. It must be admitted, however, that the experimental evidence $(27.51)$ that a higher $\dot{e}$ results in a smaller $p^{*}$ (sharper peak, steeper declining slope) is not very consistent.

One question which remains to be settled is that of the equivalent strain-rate magnitude to be substituted in the foregoing equations in case of triaxial rather than uniaxial behavior. The equivalent strain-rate magnitude, $\dot{e}$, must be given in terms of strain-rate invariants and must be always nonnegative. There are two possibilities, namely to take $\dot{e}$ as the rate of the strain invariant or as the invariant of the strain rate. The former possibility does not appear to be reasonable when 
we consider changes of strain at a constant value of the strain invariant. This is because the strain components could vary at consistent $\dot{e}$. Moreover, the rate of an invariant can be negative, while our previous equations allow only nonnegative values of $\dot{e}$. So we must adopt the second possibility.

Because concrete exhibits deviatoric as well as volumetric creep, $\dot{e}$ should depend on both the first and second invariants of $\dot{\epsilon}_{i j}$. Then, neglecting the possible influence of the third invariant of $\dot{\epsilon}_{i j}$, we may define the strain-rate magnitude as

$$
\dot{e}=\left[A\left(\frac{1}{2} \dot{e}_{i j} \dot{e}_{i j}\right)+B\left(\dot{\epsilon}_{k k}\right)^{2}\right]^{1 / 2}
$$

in which $\dot{e}_{i j}=$ deviator of strain-rate tensor $\dot{\boldsymbol{\epsilon}}_{i j}$. Note that the expression $\dot{e}=$ $A\left(\dot{e}_{i j} \dot{e}_{i j} / 2\right)^{i / 2}+B \dot{\epsilon}_{k k}$ would be inappropriate because, unlike Eq. 21 , it could have negative values. Replacing here $\dot{\epsilon}_{k k}$ by $\left|\dot{\epsilon}_{k k}\right|$ would guarantee positive $\dot{e}$, but the expression for $\dot{e}$ would not be continuously differentiable at $\dot{e}=0$, which we want to avoid. This leaves us with $\mathrm{Eq} .21$ as the most reasonable choice.

In the absence of experimental information, we will choose $A$ and $B$ such that Eq. 21 simplifies to $\dot{e}=\left(A \dot{\epsilon}_{i j} \dot{\epsilon}_{i j}\right)^{1 / 2}$; this happens for $B / A=2 / 3$. Coefficient $A$ should further be chosen such that $\dot{e}$ reduces to $\left|\dot{\epsilon}_{11}\right|$ for the uniaxial test. Noting that only rather crude values of $\dot{e}$, correct merely in their order of magnitude. need be known for practical purposes, it seems sufficient for us to assume that the lateral strain rates in the uniaxial test are constant and given by $\dot{\epsilon}_{22}=\dot{\epsilon}_{33}$ $=-\dot{\epsilon}_{11} / 3$. This is a compromise between the value $-\dot{\epsilon}_{22} / \dot{\epsilon}_{11} \simeq 0.2$ applicable in the elastic range, and values over 0.5 when $\sigma_{p}$ is approached. Evaluating Eq. 21 for these values of $\dot{\epsilon}_{11}, \dot{\epsilon}_{22}, \dot{\epsilon}_{33}$ we find that $\dot{e}$ is equal to $\dot{\epsilon}_{11}$ when $A=0.904^{2}$. Therefore, it is reasonable to define the strain-rate magnitude as

$$
\dot{e} \simeq 0.9 \sqrt{\dot{\epsilon}_{i j} \dot{\epsilon}_{i j}}
$$

However, coefficients $A$ and $B$ in Eq. 21, especially their possible dependence on the stress and strain invariants, should be re-examined when triaxial tests become available.

The triaxial constitutive relation at constant strain rate is now defined by Eqs. 10 or 11 with $a, b$, and $p^{*}$ given by Eqs. 13 , and 16-20, and the equivalent strain rate by $\mathrm{Eq}$. 22 . For the plastic-fracturing theory, the constitutive relation (Eq. 2) thus assumes the general form

$$
\dot{\boldsymbol{\sigma}}_{i j}=C_{i, k m}(\boldsymbol{\sigma}, \mathbf{\epsilon}, \dot{e}) \dot{\boldsymbol{\epsilon}}_{k m}
$$

Comparison with Tests. - The rate-independent stress-strain relation for plastic-fracturing theory (Eq. 2) was determined (12) from test data that had been obtained at strain rates of one and the same order of magnitude, roughly $10^{-5} /$ sec. The present rate-dependent generalization enabled us to fit the existing test data, as shown by the solid curves in Figs. 3-5. These curves were calculated by a computer program based on Ref. 12, which integrates the three-dimensional constitutive equations of plastic-fracturing theory in an incremental manner. The values of strength have been obtained as the peak points of the calculated curves. Instead of replacing $\epsilon_{i j}$ and $\sigma_{i j}$ with a $\epsilon_{i j}$ and $b \sigma_{i j}$ wherever $\epsilon_{i j}$ and $\sigma_{i j}$ appear in the program, the coefficients $a$ and $b$ have been combined with the material parameters as described in Appendix 1 .
About an equally good description of the test data in Figs. 3-5 could be obtained by introducing parameters $a, b$, and $p^{*}$ into the endochronic theory $(6,9,10,18,19)$

Fig. 2(a) shows the fits of the complete uniaxial stress-strain curves obtained for different strain rates by Dilger (27). It shows that a higher strain rate, the peak of stress becomes sharper. Fig. 2(b) gives the effect of strain rate on the strain at peak stress according to different investigators. Although this effect is quite mild, the experimental results obtained by different authors are contradictory; some indicate an increase of the strain, $\epsilon_{p}$, at peak stress and some a decrease as $\dot{e}$ increases. Our theory gives a slight increase of $\epsilon_{p}$ with increasing strain rate. Fig. 2(c) displays the measurements of the strength gain due to an increase of the strain rate, as obtained by various investigators, and Fig. 2(d) shows similar data for the increase of secant elastic modulus.

Fig. 3(a-c) shows the effect of strain rate on the stress at various strains. Fig. $3(d-f)$ shows that the effect of confinement due to transverse reinforcement is about the same for various strain rates. This effect was calculated by imposing the conditions of compatibility between the triaxial nonlinear deformation of concrete and the elastic-plastic deformation of reinforcement. The calculation was made in the same manner as described in Ref. 12.

Applicability in Dynamic Problems.-Although the formulation in Eq. 23 (or Eqs. 11 and 16-20) has been derived on the basis of test results at constant
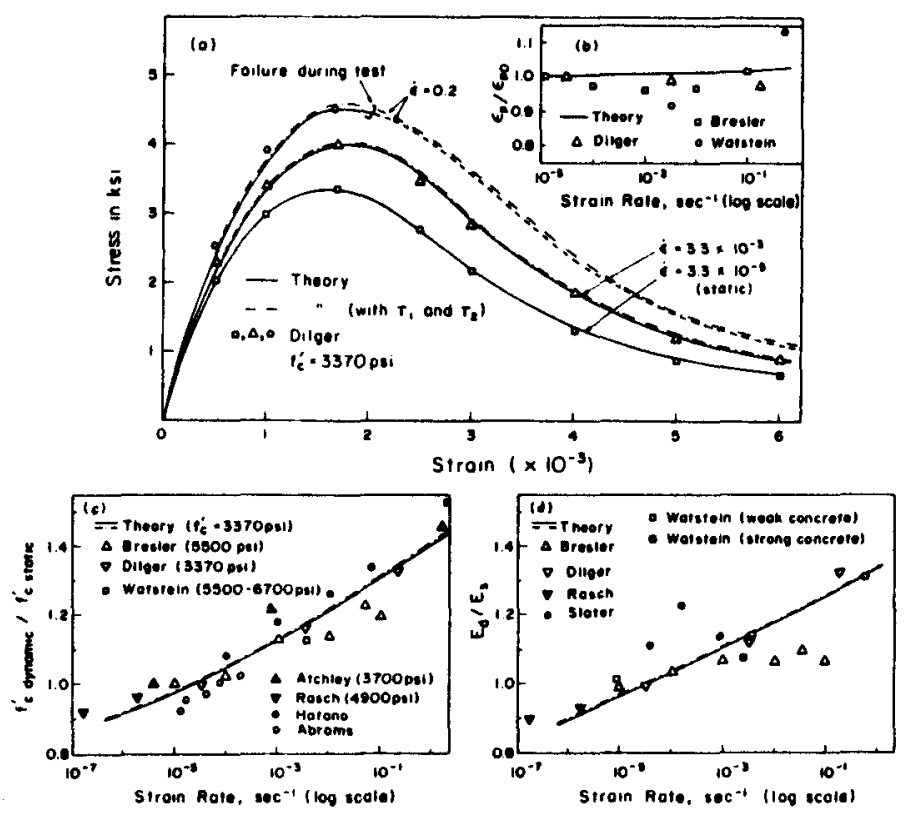

FIG. 2.-(a) Stress-Strain Relationship for Plain Concrete under Various Strain Rates; (b) Eftect of Strain Rate on Strain at Moximum Stress; Effoct of Strain Rate (c) on Compressive Strength of Plain Concrote; $(d)$ on Secant Modulus of Elastioity $\left[E_{\text {sec }}\right.$ at $\sigma=$ $0.45 f_{c}^{\prime}$ (Brester) or $\epsilon=0.001$ (others) $\left(1 \mathrm{psl}=6,895 \mathrm{~N} / \mathrm{m}^{2}\right.$ ) 
strain rate, it may be applied in a computer program for dynamic structural analysis in which the strain rate varies. In such a case, one substitutes for $\dot{e}$ in $\mathrm{Eq}$. 23 a certain characteristic value of strain rate estimated in advance, eventually iterating the analysis with improved estimates of $\dot{e}$. This is in accordance with the widespread and well established practice for metals (45).

Alternatively, one might be tempted to substitute in Eq. 23 the current value of $\dot{e}$; this, however, makes the incremental stress-strain relation nonlinear. If one tries to avoid this nonlinearity by substituting the value of $\dot{e}$ from the preceding step, the solution may become numerically unstable.

The use of Eq. 23 for dynamic problems with variable strain rate is, however, only approximate. The error seems to be acceptable when dealing with situations
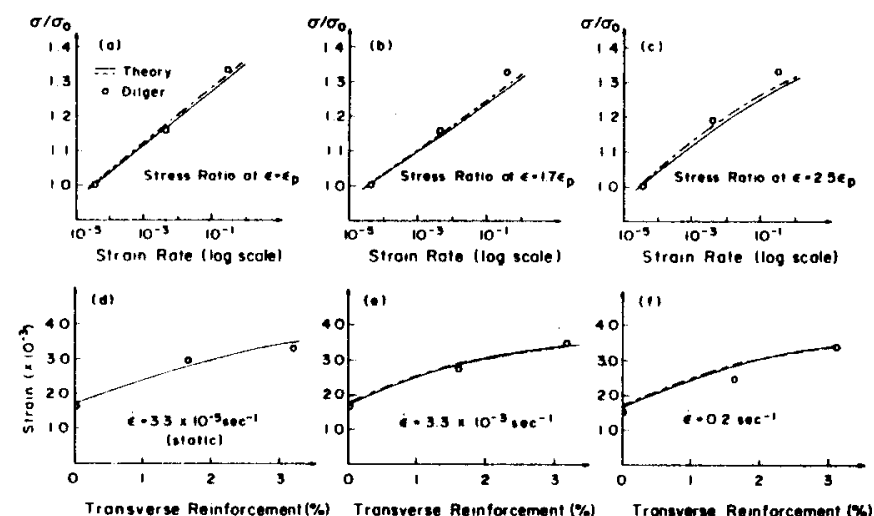

FIG. 3.-(a-c) Effect of Strain Rate on the Stress at Various Strains for Confined Concrete $(S=1$ in. $=25.4 \mathrm{~mm}) ;(d-f)$ Strain at Maximum Stress for Difforent Ratlos of Reinforcement $\left[f_{i}^{\prime}=3,370 \mathrm{psi}\left(1 \mathrm{psi}=6,895 \mathrm{~N} / \mathrm{m}^{2}\right)\right]$
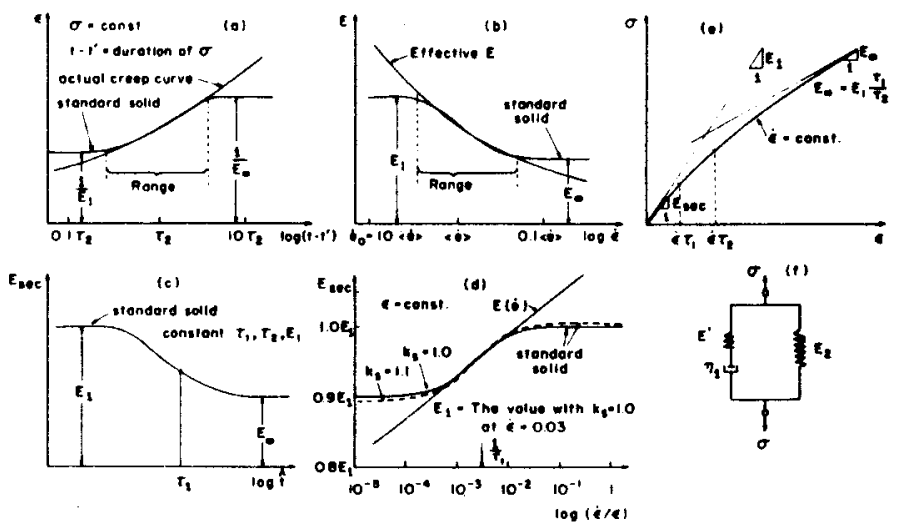

FIG. 4. - Response of Stendard Solid Compared to (a) Actual Croop Curve and to (b) Actual Function, $E(e)$; (c) Relaxation Curve of Standard Solid; (d) Adjustment of Standard Solid to Function $E(e) ;(e)$ Standard Solid Response at $\dot{\epsilon}=$ constant; $(f)$ Standard Solid Model
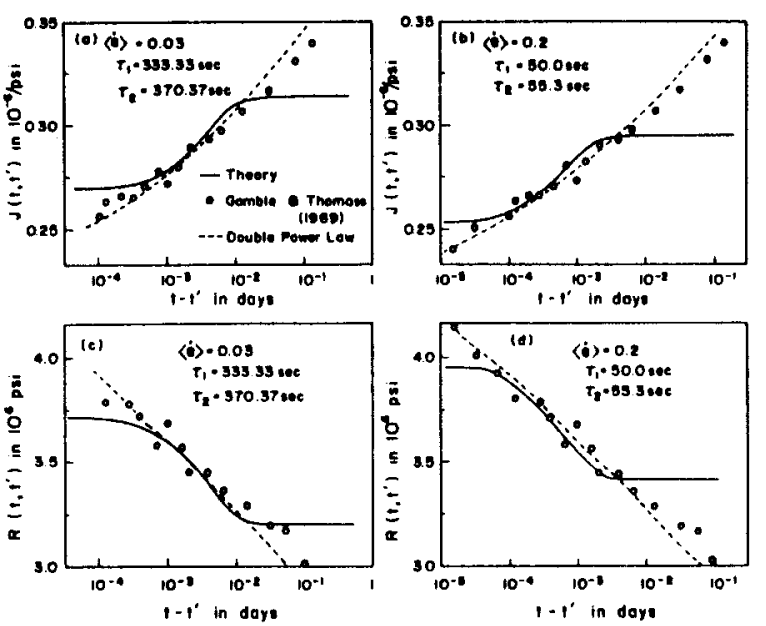

FIG. 5.-Comparison of: $(a, b)$ Short-time Creep; $(c, d)$ Short-time Relaxation (1 psi = $6,895 \mathrm{~N} / \mathrm{m}^{2}$ )

where the structure is constantly in motion, as in vibrations, blast, impact, or seismic loading. However, the method becomes inapplicable when the order of magnitude of strain rate greatly differs for various time intervals. For example, when the strain is rapidly applied and is then held constant for a long time $\left(\dot{\boldsymbol{\epsilon}}_{i j}\right.$ $=0$ ), as in relaxation tests, Eq. 23 yields constant stress $\left(\dot{\sigma}_{i j}=0\right)$. Conversely, when the stress is rapidly applied and is then held constant for a long time $\left(\dot{\sigma}_{i j}=0\right)$, Eq. 23, in general, yields a constant strain $\left(\dot{\epsilon}_{i j}=0\right)$.

So we see that our model is incapable of representing stress relaxation and creep. This limitation is not insignificant for all dynamic loads $(7,8,11,14,15$, $22,43,44,47)$, since measurements indicate significant rapid creep even for load durations under $1 \sec (29,64)$. Therefore, we will now propose a generalization that is capable of exhibiting all important viscoelastic phenomena.

\section{Inconpopution of Viscoelastic Effects}

We will now seek to generalize Eq. 23 so as to model the basic viscoelastic effects such as creep and stress relaxation. First we consider the simplest, uniaxial case. The uniaxial elastic relation $\dot{\sigma}=E \dot{\epsilon}$, has, as its most general viscoelastic counterpart, the relations $\mathscr{P \sigma}=E 2 \epsilon$, in which $\mathscr{P}$ and 2 are differential operators, and $\mathscr{P}=1+\mathrm{P}_{1} \partial / \partial t+\mathrm{P}_{2} \partial^{2} / \partial t^{2}+\mathrm{P}_{3} \partial^{3} / \partial t^{3}+\ldots, 2=$ $1+Q_{1} \partial / \partial t+Q_{2} \partial^{2} / \partial t^{2}+\ldots$ To keep practical applications simple, we will consider only differential operators of the first order; $\mathscr{P}=1+P_{1} \partial / \partial t$, and 2 $=1+Q_{1} \partial / \partial t$. For these

$\dot{\sigma}_{i j}+\frac{1}{\tau_{1}} \sigma_{i j}=C_{i j k m}^{e}\left(\dot{\epsilon}_{k m}+\frac{1}{\tau_{2}} \epsilon_{k m}\right)$

in which, in contrast to Eq. 23, moduli $C_{i j \mathrm{~m}}^{e}$ are independent of the strain rate, and coefficients $\tau_{1}$ and $\tau_{2}$ have dimensions of time and may be called the relax- 
ation time and the retardation time, respectively. In Eq. 24, we assume that $\tau_{\text {t }}$ as well as $\tau_{2}$ is the same for all tensorial components. Possibly, one value of $\tau_{1}$ might apply for the deviatoric deformation and another one for the volumetric deformation. However, no test data are available in this regard for rapid loading. For low-stress long-time creep, though, the test data show that creep Poisson ratio is constant, and thus they lend support to this assumption.

In determining the coefficients in Eq. 24, we must replace the current strainrate magnitude, $\dot{e}$, with some fixed value, $\langle\dot{e}\rangle$, characteristic of the given structural dynamics problem. We define it in the root-mean-square sense:

$\langle\dot{e}\rangle=\left(\frac{1}{V T_{1}} \int_{V} \int_{T_{1}}(\dot{e})^{2} d t d V\right)^{1 / 2}$

in which $T_{1}=$ the time interval for which the dynamic effects are to be studied; and $V=$ the volume of the body. Before starting the inelastic analysis, $\langle\dot{e}\rangle$ can be estimated from Eq. 25 on the basis of an elastic solution of the problem. After the inelastic analysis, Eq. 25 may be used to get an improved estimate of $\langle\dot{e}\rangle$, and the whole inelastic analysis may be iterated.

A fixed value, $\langle\dot{e}\rangle$, has to be used in Eq. 25 for the following reasons. For low stresses, moduli $C_{i, k m}^{2}$ in Eq. 24 are constant, i.e., independent not only of $\dot{e}$ but also of the stress and strain. Then $\mathrm{Eq}$. 24 represents the standard viscoelastic solid (Fig. $4(f)$ ), which exhibits viscoelastic phenomena within only a relatively narrow range of load durations, as shown by the creep curves in Fig. 4(a), and within a relatively narrow range of strain rates. This range spans over one order magnitude (Fig. $4(b)$ ), roughly from $0.3\langle\dot{e}\rangle$ to $3\langle\dot{e}\rangle$ if $\langle\dot{e}\rangle$ is chosen to characterize the middle of the range. For the strain rate $10\langle\dot{e}\rangle$, the behavior of the standard solid is then essentially elastic, the elastic modulus being equal to the instantaneous modulus of the standard solid ( $E_{1}$ in Fig. 4). For the strain rates below $0.1\langle\dot{e}\rangle$, the behavior is also essentially elastic, but with the elastic modulus equal to the long-time modulus of the standard solid ( $E_{x}$ in Fig. 4). Within the range $0.3\langle\dot{e}\rangle$ to $3\langle\dot{e}\rangle$, the relaxation and retardation times in Eq. 24 are capable of characterizing all viscoelastic effects, including not only creep and stress relaxation but also the strain-rate effect. Consequently, $C_{i j \mathrm{~km}}^{e}$ cannot be considered to depend on the current strain rate, $\dot{e}$, or else the strain-rate effect would incorrectly be obtained twice-first from $C_{i, k m}^{r}$ and second from $\tau_{1}$ and $\tau_{2}$.

It should be noted that the characteristic strain rate, $\langle\dot{e}\rangle$, as defined by Eq. 25 , depends on the nature of dynamic loading and the geometry of the structure. This might seem to put in question the predictive capability of the extended model with viscoelastic effects which we are about to develop in the rest of this paper. We should realize, however, that $\langle\dot{e}\rangle$ needs to be known only within an order of magnitude, i.e., it can be five times smaller or larger than the correct value. An order of magnitude estimate of $\langle\dot{e}\rangle$ can be easily achieved in advance, for example by carrying out an elastic dynamic analysis. So the introduction of $\langle\dot{e}\rangle$ does not deprive our method of its predictive capability.

The need for an a priori estimation of $\langle\dot{e}\rangle$ could be removed by using a general viscoelastic model corresponding to a Maxwell or Kelvin chain with a broad range of relaxation and retardation times spanning over many orders of magnitude. This, however, would greatly complicate the structural analysis, much more so than the a priori estimation of $\langle\dot{e}\rangle$. It would then be necessary to store for each finite element a number of internal variables (partial stresses or partial strains) and use special complicated time-step integration formulas that assure stability even when the time step is much larger than some of the relaxation times (5). The method of such analysis is, in general, known and is the same as for longtime creep (5), but it seems unnecessarily complicated for dynamic problems in which only the viscoelastic phenomena corresponding to a rather limited range of relaxation times are of importance. It has been for the purpose of avoiding these complications that the simple expedient of strain-rate dependent elastic moduli (Eq. 23) has been used in the past. To avoid the main limitations of this approach, we now propose the use of $\langle\dot{e}\rangle$ as the best compromise between simplicity (no internal variables) and generality (representation of all viscoelastic effects).

Now we try to determine from the strain-rate dependence of $C_{\text {iktm }}$ (Eq. 23) the relaxation and retardation times, $\tau_{1}$ and $\tau_{2}$, necessary to provide about the same strain-rate effect as that we lost by replacing the strain-rate dependent elastic moduli $C_{i j t m}$, by the fixed ones, $C_{i j k m}^{e}$. Noting that the uniaxial standard solid response is characterized by the constitutive relation, $\dot{\sigma}+\sigma / \tau_{1}=E_{1}(\dot{\epsilon}+$ $\epsilon / \tau_{2}$ ), in which $E_{1}=$ instantaneous elastic modulus of the standard solid (Fig. $4(f)$ ), we obtain, for constant strain rate $\dot{\epsilon}$ (see Fig. $4(c, d, e)$ )

$\sigma=E_{\text {sec }} \epsilon ; \quad E_{\text {sec }}=E_{1}\left[\frac{\tau_{1}}{\tau_{2}}+\left(1-\frac{\tau_{1}}{\tau_{2}}\right) \frac{\tau_{1} \dot{\epsilon}}{\epsilon}\left(1-e^{-\epsilon / \tau_{1}}\right)\right] \ldots \ldots \ldots \ldots$

in which $\epsilon / \dot{\epsilon}=\hat{t}=$ time measured from the start of deformation. For large $\dot{\epsilon}$ $(\dot{\epsilon} \rightarrow \infty)$, we may set $\left(1-e^{-\epsilon / \epsilon \tau_{1}}\right) \tau_{1} \dot{\epsilon} / \epsilon=1-\epsilon / 2 \dot{\epsilon} \tau_{1}$.

The point $\hat{t}=\tau_{1}$ is approximately (in the log-time scale) in the middle of the single-decade time range in which the standard solid behaves viscoelastically. We must now match the behavior of the standard solid to that given by function $E(\dot{e})\left(\mathrm{Eq} .16\right.$ or Eq. 20). For the matching point, we choose $i=\tau_{1}$. By the same argument as the one used to obtain Eq. 15, the proper choice is

$\tau_{1}=(0.1\langle\dot{e}\rangle)^{-1}$ (seconds)

To determine the remaining two parameters, $\tau_{2}$ and $E_{1}$, our matching conditions are that for $\hat{i}=\tau_{1}$ and for $\dot{e}=\langle\dot{e}\rangle$, the modulus value in Eq. 26 for the standard solid must coincide with the value, $E(\dot{e})$, obtained from the constant-strain-rate test (Fig. $4(d)$ ), and that the slope, $\partial E_{\text {sec }} / \partial \dot{\epsilon}$, according to Eq. 26, must agree with the slope of the given curve, $E(\dot{e})$ :

$E_{\text {sec }}=E(\dot{e}) ;\left[\frac{\partial E_{\text {sec }}}{\partial \dot{\epsilon}}\right]_{e=\text { constant }}=k_{s} \frac{d E(\dot{e})}{d \dot{e}}$

The value $k_{s}=1$ gives obviously the best fit near the matching point, but for the value $k_{s}=1.1$, the fit near the matching point is still acceptable and the range of good fit gets wider (see Fig. $4(d)$ ). Thus, $k_{s}=1.1$ is a preferable choice. Now, substituting here Eq. 26, we have the conditions

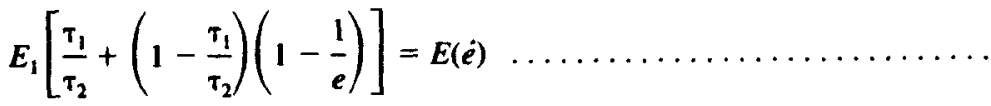


and $\left(1-\frac{2}{e}\right) \frac{E_{1}}{\dot{\epsilon}}\left(1-\frac{T_{1}}{\tau_{2}}\right)=k_{s} \frac{d E(\dot{e})}{d \dot{e}} \ldots$

Setting $\dot{\epsilon}=\dot{e}, e=2.718$, and solving these equations, we obtain

$E_{1}=E(\dot{e})+1.39 k_{\mathrm{s}} \dot{e} \frac{d E(\dot{e})}{d \dot{e}} \quad($ for $\dot{e}=\langle\dot{e}\rangle$

$\tau_{2}=\frac{\tau_{1} E_{1}}{2.72 E(\dot{e})-1.72 E_{1}}$

Note that always $E_{1}>E(\dot{e})$ and $\tau_{2}>\tau_{1}$

As for the strain-rate independent moduli in Eq. 24, $C_{i, k m}^{e}$, we assume them to be all proportional to modulus $E_{1}$. This assumption is correct if the creep Poisson ratio is constant for rapid creep, which seems likely since it is so for the long-time creep. Thus, we may use in Eq. 24

$C_{i j k m}^{e}=\frac{E_{1}}{E(\langle\dot{e}\rangle)} C_{i j k m}(\boldsymbol{\sigma}, \epsilon,\langle\dot{e}\rangle)$

$=\left\{1+1.39\left[\frac{k_{s} \dot{e}}{E(\dot{e})} \frac{d E(\dot{e})}{d \dot{e}}\right]_{i=\langle\dot{e})}\right\} C_{i j k m}(\boldsymbol{\sigma}, \epsilon,\langle\dot{e}\rangle)$

in which moduli $C_{i j k m}$ are those which correspond to a constant strain rate. $\langle\dot{e}\rangle$ (Eq. 23)

Integration and Comparison with Tests.-To integrate Eq. 24 with elastic moduli according to Eq. 33, a step-by-step algorithm was programmed. The tangential moduli, $C_{i j k m}$, from $\mathrm{Eq} .33$, also used in $\mathrm{Eq} .23$, were determined according to the plastic-fracturing theory (Eq. 21 of Ref. 12). Several previously published sets of test data $(1,2,23,27,35,51,63,64)$, the same as those we already fitted by Eq. 23 (Figs. 2-3), were fitted again. The fits are shown as the dashdot curves in Figs. 2-3. The material parameters are listed in Appendix I.

To demonstrate that the present model is capable of describing creep at constant stress and stress relaxation at constant strain, the calculated creep and relaxation curves are compared in Fig. 5 to the test data from Refs. 16, 17, and 29. For tests at constant strain rate, the model (Eqs. 24, 33) is equivalent to our previous model with strain-rate dependent elastic moduli (Figs. 2-3, 5).

In calculating the fits of the data at constant stress or constant strain, it was assumed for the sake of simplicity that within the range shown in these figures the variation of $C_{i j k m}$ as functions of $\sigma$ and $\epsilon$ is not very significant because of the relatively low stress level $\left(\approx 0.3 f_{c}^{\prime}\right)$. This makes it possible to consider $C_{i j k m}$ as constants (54). Since $\tau_{1}$ and $\tau_{2}$ are constants (determined from Eqs. 27 and 32 on the basis of fixed mean strain rate $(\dot{e}))$, we may write the solution of Eq. 24 in a closed form:

For $\dot{\sigma}=0$ (creep): $\quad \epsilon=C^{-1} \sigma \frac{\tau_{2}}{\tau_{1}}-\left[C^{-1} \sigma \frac{\tau_{2}}{\tau_{1}}-\epsilon\left(t_{o}\right)\right] e^{-t / \tau_{2}}$

For $\dot{\epsilon}=0$ (relaxation):

$$
\sigma=C \epsilon \frac{\tau_{1}}{\tau_{2}}+\left[\sigma\left(t_{o}\right)-\operatorname{Ce} \frac{\tau_{1}}{\tau_{2}}\right] e^{-t_{1 / \tau}}
$$

in which $\sigma$ and $\epsilon$ are $(6 \times 1)$ column matrices of stress and strain components; and $C$ is the $(6 \times 6)$ matrix of the moduli, $C_{i j \mathrm{~km}}$. Matrices $C$, $\sigma$, and $\epsilon$ at the right-hand sides of these equations were obtained by using the previously developed (12) computer program for the plastic-fracturing theory for the rapid loading up to the start $\left(t=t_{o}\right)$ of creep $(\dot{\boldsymbol{\sigma}}=0)$ or relaxation $(\dot{\boldsymbol{\epsilon}}=0)$.

Since no relaxation test data are available for the very rapid short-time relaxation, the data points in Fig. 5 were obtained from the approximate formula $R\left(t, t^{\prime}\right) \approx 1 / J\left(t, t^{\prime}\right)$, in which $R\left(t, t^{\prime}\right)$ is the relaxation function equal to the stress at time $t$ due to a unit strain imposed at time $t^{\prime}$.

Behavior at Unloading and Hysteresis.-The special uniaxial case of Eq. 24 may be written as

$\dot{\boldsymbol{\sigma}}-\boldsymbol{E} \dot{\epsilon}=f(\sigma, \epsilon)$

in which $f(\sigma, \epsilon)=E \epsilon / \tau_{2}-\sigma / \tau_{1}=$ function of $\sigma$ and $\epsilon$. An equation of this type has been used $(26,40)$ to model the irreversible behavior of the viscoplastic metals at loading as well as unloading; it permits modeling the unloading and hysteresis at cyclic loading without the use of any additional loading-unloading criteria. Consider a state point $(\sigma, \epsilon)$ at which the loading reverses to unloading. Let the superscripts minus denote the variables just before the reversal point, and the superscript plus denote the variables just after this point. Then we have $\dot{\sigma}^{+}-E \dot{\epsilon}^{+}=f(\sigma, \epsilon)$ and $\dot{\sigma}^{-}-E \dot{\epsilon}^{-}=f(\sigma, \epsilon)$. Subtracting these equations we get

$\dot{\boldsymbol{\sigma}}^{+}-\dot{\boldsymbol{\sigma}}^{-}=E\left(\dot{\boldsymbol{\epsilon}}^{+}-\dot{\boldsymbol{\epsilon}}^{-}\right)$

Here we may set $\dot{\sigma}^{+}=\left(d \sigma^{+} / d \epsilon\right) \dot{\epsilon}^{+}$and $\dot{\sigma}^{-}=\left(d \sigma^{-} / d \epsilon\right) \dot{\epsilon}^{-}$. If the strain-rate magnitude remains the same, i.e., $\dot{\epsilon}^{+}=-\dot{\boldsymbol{\epsilon}}^{-}$, Eq. 37 becomes

$\frac{1}{2}\left(\frac{d \sigma^{+}}{d \epsilon}+\frac{d \sigma^{-}}{d \epsilon}\right)=E$

This means that the average of the loading and unloading slopes equals the elastic slope, $E$. Thus, upon a reversal to unloading, there is a sudden change in the slope of the stress-strain diagram. This is shown in Fig. 6(a).

Alternatively, we may also set in Eq. $37 \dot{\epsilon}^{+}=(d \epsilon+d \sigma) \dot{\sigma}^{+}, \dot{\epsilon}^{-}=\left(d \epsilon^{-} / d \sigma\right)$ $\dot{\sigma}^{-}$. If the stress-rate magnitude remains the same, i.e. $\dot{\boldsymbol{\sigma}}^{+}=-\dot{\boldsymbol{\sigma}}^{-}, \mathrm{Eq} .36$ becomes

$\frac{1}{2}\left(\frac{d \epsilon^{+}}{d \sigma}+\frac{d \epsilon^{-}}{d \sigma}\right)=\frac{1}{E}$
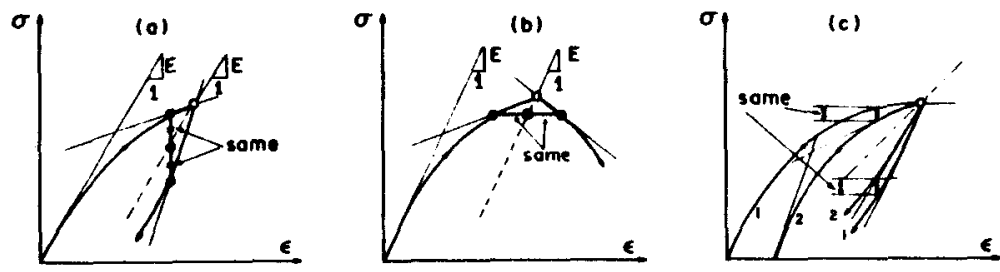

FIG. 6.-Slopes for Unloading: (a) Same $|\dot{k}| ;$ (b) Same $|\dot{o}| ;$ (c) Same $|\dot{k}|$ 
This gives the change of slope depicted in Fig. $6(b)$.

As another case, consider that $\left|\dot{\boldsymbol{\epsilon}}^{+}\right|$is much larger than $\left|\dot{\boldsymbol{\epsilon}}^{-}\right|$, i.e. $\left(-\dot{\boldsymbol{\epsilon}}^{+} / \dot{\boldsymbol{\epsilon}}^{-}\right)$ $\rightarrow \infty$. Dividing Eq. 37 by $\dot{\epsilon}^{+}$we then find that $d \sigma^{+} / d \epsilon=E$, i.e. the unloading slope is elastic, as could have been expected.

The plastic-fracturing theory, as well as the endochronic theory, exhibits a change of slope at unloading even without any strain-rate (or time) effects. The question is: How are these effects modified for the present time-dependent generalization? The time-independent versions of these theories $(6,7,9,10,12,18,19)$ are characterized by a certain tangent modulus, $E_{t}$, for loading, and the unloading slope is at the beginning elastic, i.e., $E^{-}=E_{1}$, and $E^{+}=E$ for the time-independent versions. So, $\dot{\sigma}^{+}-E \dot{\epsilon}^{+}=f(\sigma, \epsilon)$, and $\dot{\sigma}^{-}-E, \dot{\epsilon}^{-}=f(\sigma, \epsilon)$. Subtracting these two equations, we obtain, instead of Eq. 37

$\dot{\boldsymbol{\sigma}}^{+}-\dot{\boldsymbol{\sigma}}^{-}=E \dot{\boldsymbol{\epsilon}}^{+}-E_{\imath} \dot{\boldsymbol{\epsilon}}^{-}$

Now, if the strain-rate magnitude remains the same, $\dot{\epsilon}^{+}=-\dot{\epsilon}^{-}$, and Eq. 40 yields

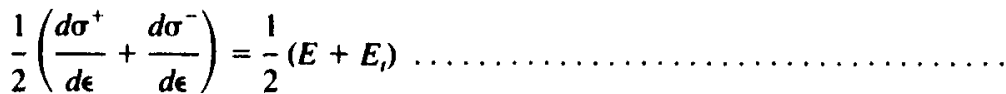

i.e., the average of the loading and unloading slopes with and without the time (strain-rate) dependence is (for the present theory) the same. This is graphically shown in Fig. 6(c). Although no good test data for unloading at various high strain rates appear to exist, the behavior shown in Fig. 6(c) seems to be reasonable.

Thus, the introduction of time-dependent (strain-rate) effects into the nonlinear triaxial theories for concrete intensifies the irreversibility at unloading, and consequently also the hysteresis at cyclic loading. Since, however, these theories were fitted in Ref. 9 or 12 to unloading data without consideration of the rate effect, the unloading irreversibility with the present generalization might become too strong and readjustment might be needed.

\section{Summary and Conclusions}

Presented is a generalization of time-independent nonlinear triaxial constitutive relations for concrete to model short-time viscoelastic effects. Carrying out affinity and shape transformations of the original time-independent constitutive equation, one can introduce in any constitutive model the effect of strain-rate magnitude on the initial elastic modulus, the peak stress (strength), and the sharpness of the stress peak (related to the post-peak declining slope). The method is calibrated by numerically fitting test data from the literature spanning over many orders of strain-rate magnitude. The fits are, in particular, demonstrated for a generalization of the plastic-fracturing theory for concrete.

To obtain a model that also exhibits short-time rapid creep and stress relaxation, the incrementally linear constitutive law characterized by tangential moduli as functions of the invariants of stress, strain, and strain rate is replaced by a tensorial first-order differential equation in stress, strain, stress rate, and strain rate. Its special linear case may be regarded as the standard solid, characterized by the relaxation time, the retardation time and the (apparent) instantaneous modulus independent of the strain rate. These three parameters are determined by requiring that this formulation and the original strain-rate dependent formulation coincide for tests at constant strain rates near a certain given strain-rate magnitude which is characteristic of the structural dynamics problem at hand. Good fits of test data available in the literature are also demonstrated. Finally, the irreversibility at unloading caused by the short-time viscoelastic effects is analyzed.

The model allows more realistic dynamic finite element analysis of concrete structures. However, since nearly all available test data on the strain-rate effect are uniaxial, and even the uniaxial ones are not unambiguous (e.g., with regard to the delining slope as a function of the strain rate), revisions in the model may be expected in the future. Therefore, the model is made capable of easy adjustment and independent of the particular triaxial formulation per se.

\section{Acknomledement}

Financial support under National Science Foundation Grant ENG-14848A01 is gratefully acknowledged. The initial work on this formulation was carried out under Guggenheim Fellowship awarded to the first writer for 1978-79.

\section{Appendix. I.-Transformed Matemal Parameters}

We replace $f_{c}^{\prime}$ with $f_{1}\left(p^{*}\right), \epsilon_{i j}$ with $a \epsilon_{i j}$, and $\sigma_{i j}$ with $b \sigma_{i j}$. This replacement must include all invariants, e.g. $I_{1}$ must be replaced with $b I_{1}, J_{2}$ with $b^{2} J_{2}, J_{2}^{*}$ with $a^{2} J_{2}^{*}$, etc. Then, combining coefficients $a$ and $b$ with the material parameters given by Eqs. 30-34 of Ref. 12 (with subsequent Errata), the expressions for material parameters become functions of $a$ and $b$ as follows: $\bar{a}_{0}=\left|f_{1}\left(p^{*}\right)\right|^{4} / 90$ psi; $\tilde{a}_{1}=b\left[f_{1}\left(p^{*}\right)\right]^{3} / 150 \mathrm{psi} ; \bar{a}_{2}=b^{0.75}\left[15 \times 10^{7}(\mathrm{psi})^{2} / f_{1}\left(p^{*}\right)\right]^{2} ; \bar{a}_{3}=b^{2.75}[35,000$ $\left.\mathrm{psi} / f_{1}\left(p^{*}\right)\right]^{3} ; \bar{a}_{4}=b^{3}\left\{f_{1}\left(p^{*}\right) / 2,700 \mathrm{psi}\right]^{2} ; \bar{a}_{5}=1.95 b^{2} ; \bar{a}_{6}=1.73 b ; \bar{b}_{0}=(2 \mathrm{psi})^{2}$ $\bar{b}_{1}=36,000 b^{2} ; \bar{b}_{2}=\left(13 \times 10^{4}\right) b^{2} ; \bar{b}_{3}=b\left[14,000 \mathrm{psi} / f_{1}\left(p^{*}\right)\right\} ; \bar{b}_{4}=134 b^{2} ;$ $\bar{b}_{3}=b^{3}\left[45,000 \mathrm{psi} / f_{1}\left(p^{*}\right)\right]^{2.5} / \mathrm{psi} ; \bar{b}_{6}=a b^{3}\left[f_{1}\left(p^{*}\right) / 840 \mathrm{psi}\right]^{5} / \mathrm{psi} ; \bar{c}_{0}=(96 \times$ $\left.\left.10^{5}\right) a^{2} b^{2} ; \bar{c}_{1}=a^{2} b^{2}\left(405 \times 10^{5}\right) ; \bar{c}_{2}=4,650 \mathrm{psi} ; \bar{c}_{3}=b \mid 14,000 \mathrm{psi} / f_{1}\left(p^{*}\right)\right] ; \bar{c}_{4}$ $=b^{2}\left[f_{1}\left(p^{*}\right) / 1,350 \mathrm{psi}\right]^{6} ; \vec{c}_{5}=110 b^{2} ; \bar{\alpha}_{0}=0.5 ; \bar{\alpha}_{1}=b^{3}\left\{25,000 \mathrm{psi} /\left[f_{1}\left(p^{*}\right)\right]^{2}\right\} ;$ $\bar{\alpha}_{2}=b^{2}\left[11,000 \mathrm{psi} / f_{1}\left(p^{*}\right)\right]^{0.8} ; \bar{\alpha}_{3}=(1 \mathrm{psi})^{2} ; \bar{\alpha}_{4}=b^{2}\left[2,100 \mathrm{psi} /\left.f_{1}\left(p^{*}\right)\right|^{1.6} \times 10^{-6} ;\right.$ $\bar{\alpha}_{5}=b^{4}\left[f_{1}\left(p^{*}\right) /(5,540)^{4}(\mathrm{psi})^{3}\right]$, in which $1 \mathrm{psi}=6,895 \mathrm{~N} / \mathrm{m}^{2}$

Aside from replacing the material parameters as indicated, the incorporation of the strain rate effect requires other adjustment neither in the theory nor in the computer program described in Ref. 12.

\section{Appenolx H.-Peferences}

1. Abrams, D. A., "Effect of Rate of Application of Load on the Compressive Strength of Concrete," Proceedings of the American Sociery on Testing and Materials. Vol. 17. Part II, p. 364.

2. Atchley, B. L., and Furr, H. L., "Strength and Energy Absorption Capabilities of Plain Concrete Under Dynamic and Static Loadings," American Concrete Institute Journal, Vol. 64, No. 11, Nov., 1967, pp. 745-756.

3. Bažant, Z. P., "Numerical Determination of Long-Range Stress-History from Strain History in Concrete," Materials and Structures, Paris, France, Vol. 5, 1972, pp. 135-141. 
4. Bażant, Z. P., "Prediction of Concrete Creep Effects Using Age-Adjusted Effective Modulus Method," American Concrete Institute Journal, Vol. 69, 1972, pp. 212-217.

5. Bażant, Z. P., "Theory of Creep and Shrinkage in Concrete Structures: A Précis of Recent Developments," Mechanics Today, Pergamon Press, Inc., New York, N.Y., Vol. 2, 1975, Pp. 1-93.

6. Bažant, Z. P., "Endochronic Inelasticity and Incremental Plasticity," International Journal of Solid and Structures, Vol. 14, 1978, pp. 691-714.

7. Bažant, Z. P., "Inelasticity and Failure of Concrete: A Survey of Recent Progress," Seminar on Analysis of Reinforced Concrete Structures by Finite Element Method (Commemorating the 50th Anniversary of School of Reinforced Concrete), Politecnico di Milano, Milan, Italy, June, 1978, pp. 5-59.

8. Bazant, Z. P., and Asghari, A. A., "Constitutive Law for Nonlinear Creep of Concrete," Journal of the Engineering Mechanics Division, ASCE, Vol. 103, No. EMI Proc. Paper 12729, Feb., 1977, pp. 113-124.

9. Bažant, Z. P., and Bhat, P., "Endochronic Theory of Inelasticity and Failure of Concrete," Journal of the Engineering Mechanics Division, ASCE, Vol. 102, No. EM4, Proc. Paper 12360, Aug., 1976, pp. 701-722.

10. Bažant, Z. P., Bhat, P., and Shieh, C.-L.." "Endochronic Theory for Inelasticity and Failure Analysis of Concrete Structures." Structural Engineering Report No. 197612/259, (to Oak Ridge National Laboratory), Northwestern University, Evanston Illinois, Dec., 1976 (available from National Technical Information Service, Springfield, $\mathrm{Va}$ )

11. Bažant, Z. P., Carreira, D., and Waiser, A., "Creep and Shrinkage in Reactor Containment Shells," Journal of the Structural Division, ASCE, Vol. 101, No. ST10, Proc. Paper 11632, Oct., 1975, pp. 2117-2131.

12. Bažant, Z. P., and Kim, S.-S., "Plastic Fracturing Theory for Concrete," Journal of the Engineering Mechanics Division, ASCE, Vol. 105, No. EM3, Proc. Paper 14653, June, 1979, pp. 407-428.

13. Bažant, Z. P., Kim, S.-S., “Approximate Relaxation Function for Concrete," Journal of the Structural Division, ASCE, Vol. 105, No. ST12, Proc. Paper 15083, Dec. 1979, pp. 2695-2705.

14. Bazant, Z. P., and Kim, S.-S., "Nonlinear Creep of Concrete-Adaptation and Flow," Journal of the Engineering Mechanics Division, ASCE, Vol. 105, No. EM3, Proc. Paper 14654, June, 1979, pp. 429-446.

15. Bażant, Z. P., and Najjar, L. J., "Comparison of Approximate Linear Methods for Concrete Creep," Journal of the Structural Division. ASCE, Vol. 99, No. ST9, Proc. Paper 10006, Sept., 1973, pp. 1851-1874

16. Bażant, Z. P., and Osman, E., "Double Power Law for Basic Creep of Concrete," Materials and Structures, Paris, France, Vol. 9, 1976, pp. 3-11.

17. Bažant, Z. P., and Panula, L., "Prediction of Time-Dependent Deformations of Concrete," Materials and Structures, Paris, France, Parts I and II, Vol. 11, No. 65, 1978, pp. 307-328; Parts III and IV, Vol. 11, No. 66, 1978, pp. 415-434; Parts V 1978, Pp. 307-328; Parts III and
and VI, Vol. 12, No. 69, 1979.

18. Bazant, Z. P., and Shieh, C.-L., "Endochronic Model for Nonlinear Triaxial Behavior of Concrete," Nuclear Engineering and Design, Vol. 47, 1978, pp. 305-315.

9. Bażant, Z. P., and Shieh, C.-L., "Hysteretic Fracturing Endochronic Theory for Concrete," Journal of the Engineering Mechanics Division, ASCE, Vol. 106, No. EM5, Proc. Paper 5781, 1980, pp. 929-950.

20. Bicanic, N., Hinton, E., Pandé, G. N. and Zienkiewicz, O. C., "Nonlinear Seismic Response of Concrete Gravity Dams,", The 6-th European Conference on Earthquake Engineering, Dubrovnik, Yugoslavia, Sept., 1978.

21. Birkimer, D. L., and Lindemann, R. L., "Dynamic Tensile Strength of Concrete Materials," American Concrete Institute Journal, Jan., 1971, pp. 47-49.

22. Branson, D. E., and Christiason, M. L., "Time-Dependent Concrete Properties Related to Design-Strength and Elastic Properties, Creep and Shrinkage," ACl Special Publication SP-27, American Concrete Institute, Detroit, Mich., Paper No. 13, 1971. pp. 257-277.
23. Bresler, B., and Bertero, V V. "Influence of High Strain Rate and Cyclic Loading on Behavior of Unconfined and Confined Concrete in Compression" Report, Division of Structural Engineering and Structural Mechanics, University of Califomia, Berkeley, Calif., 1979.

24. Burgess, W. M., "Impact Resistance of Reinforced Concrete as a Problem of Containment," Research Report No. R251, Sydney University, Sydney, Australia, Aug. 1974.

25. Carmichael, G. D. T., and Hormby, I. W., "The Strain Behavior of Concrete in Prestressed Concrete Pressure Vessels," Magazine of Concrete Research, Vol. 25, No. 82, Mar., 1973, pp. 5-16.

26. Cernocky, E. P., and Krempl, E., "A Non-linear Uniaxial Integral Constitutive Equation Incorporating Rate Effects, Creep, and Relaxation," International Journal of Non-Linear Mechanics, Vol. 14, 1979, pp. 183-203.

27. Dilger, W. H., Koch, R., and Kowalczyk, R., "Ductility of Plain and Confined Concrete under Different Strain Rates," presented at American Concrete Institute Moeting, Houston, Nov., 1978, American Concrete Institute Special Publication, Dept. of Civil Engineering, University of Calgary, Calgary, Alberta, Canada, 1978

28. Evans, R. H. "Effect of Rate of Loading on the Mechanical Properties of Some Materials," Journal of the Institution of Civil Engineers, Vol. 18, 1942, pp. 296-306.

29. Gamble, B. R., and Thomass, L. H., "The Creep of Concrete Subject to Varying Stress," Proceedings of the Australian Conference on the Mechanics of Structures and Materials, Paper No. 24, Adelaide, Australia, Aug., 1969.

30. Goldsmith, W., Polivka, M., and Yang, T., "Dynamic Behavior of Concrete," Experimental Mechanics, Vol. 6, No. 2, Feb., 1966, pp. 65-179.

31. Green, H., "Impact Strength of Concrete," Proceedings of the Institution of Civil Engineers, Vol. 28, 1964, pp. 383-396

32. Griner, G. R., Sierakowski, R. L., and Ross, C. A., "Dynamic Properties of Concrete under Impact Loading." Proceedings, 45th Symposium on Shock and Vibration Bulletin, No. 45, pp. 131-142.

33. Gupta, Y. M., and Seaman, L., "Local Response of Reinforced Cuncrete to Missile Impacts," Nuclear Engineering and Design, Vol. 45, 1978, pp. 507-514.

34. Hatano, T., "Dynamic Behavior of Concrete under Impulsive Tensile Load," Technical Report: C-6002, Central Research Institute of Electric Power Industry, Japan, 1960.

35. Hatano, T., and Tsutsumi, H., "Dynamic Compressive Deformation and Failure of Concrete under Earthquake Load," Proceedings of the 2nd World Conference on Earthquake Engineering, Science Council of Japan, Tokyo, Japan, Vol. III, 1960, pp. 1963-1978.

36. Hellesland, J., and Green, R., "A Stress and Time Dependent Strength Law for Concrete," Cement and Concrere Research, Vol. 2, 1972, pp. 261-275.

37. Hughes, B. P., and Gregory, R., "Concrete Subject to High Rates of Loading in Compression," Magazine of Concrete Research. Vol. 24, No. 78, Mar., 1972, pp. 25-36.

38. Hughes, B. P., and Watson, A. J., "Compressive Strength and Ultimate Strain of Concrete under Impact Loading," Magazine of Concrete Research, Vol. 30, No. 105, Dec., 1978, pp. 189-199.

39. Jones, P. G., and Richart, F. E., "The Effect of Testing Speed on Strength and Elastic Properties of Concrete." Proceedings of the American Society on Testing and Materials, Vol. 36, 1936, pp. 380-391.

40. Liu, M. C. M., and Krempl, E., "A Uniaxial Viscoplastic Model Based on Total Strain and Overstress," Journal of Mechanics and Physics of Solids, Vol. 27, 1979. Pp. 377-391.

41. Mainston, R. J., "Properties of Materials at High Rates of Straining or Loading," Materials and Structures, Vol. 8, No. 44, pp. 108-116.

42. McHenry, D., and Shideler, J. J., "Review of Data on Effect of Speed in Mechanical Testing of Concrete," ASTM Special Technical Publication No. 185, American Society on Testing and Materials, Philadelphia, Pa., pp. 22-82.

43. Neville, A. M., Properties of Concrete, 2nd Edition, John Wiley and Sons, Inc., New York, N.Y., 1973. 
44. Neville, A. M., and Dilger, W., Creep of Concrete: Plain, Reinforced, Prestressed, North-Holland Publishing Co., Amsterdam, The Netherlands, 1970.

45. Perrone, N., "A Mathematically Tractable Model of Strain-Hardening, Rate-Sensitive Plastic Flow," Journal of Applied Mechanics, Transactions, American Society of Mechanical Engineers, Vol. 33, Mar., 1966, p. 210.

46. Philleo, R. E. " Comparison of Results of Three Methods for Determining Young's Modulus of Elasticity," American Concrete Institute Journal, Vol. 51, 1955, pp. $461-469$.

47. Pirtz, D., “Creep Characteristics of Mass Concrete for Dworshak Dam," Report No. 65-2, Structural Engineering Laboratory, University of California, Berkeley, Calif., Oct., 1968.

48. Pozzo, E., "Rheological Model of Concrete in the Dynamic Field," Meccanica, Italy. Vol. 5, No. 2, June, 1970, pp. 143-158.

49. Price, W. H., "Factors Influencing Concrete Strength," American Concrete Institute Journal, Vol. 47, 1951, pp. 417-432.

50. Radjy, F., and Richards. C. W.. "Effect of Curing Heat Treatment History on the Dynamic Response of Cement Paste," Cement and Concrete Research, Vol. 3, 1973, pp. 7-21.

51. Rasch, C., "Stress-Strain Curves of Concrete and Stress Distribution in the Flexural Compression Zone under Constant Strain Rate," Bulletin No. 154, Berlin, Germany, 1962, Deutscher Ausschuss für Stahlbeton, (in German).

52. Read, H. E., and Maiden, C. J., "The Dynamic Behavior of Concrete," No. AD894240, National Technical Information Service, Springfield, Va., Aug., 1971.

53. Sahlin, S., et al., "The Effect of Impact Loading on Building." State-of-the-Art Report, Materials and Structures, Vol. 8, No. 44, 1974, pp. 77-130.

54. Schapery, R. A., "A Method of Viscoelastic Stress Analysis Using Elastic Solutions," Journal of the Franklin Institute, Vol. 279, 1965. pp. 268-289.

55. Sharma, M. R., and Gupta, B. L., "Sonic Modulus as Related to Strength and Static Modulus of High Strength Concrete," Indian Concrete Journal, Vol. 34, 1960, pp. 139-141.

56. Sinisalo, H. S., Tuomala, M. T. E., and Mikkola, M. J., "Nonlinear Dynamic Analysis of Reinforced Concrete Slabs," Report, Department of Civil Engineering, Helsinki University of Technology, 02150 Espoo 15, Finland, 1979.

57. Sparks, P. R., and Menzies, J. B., "The Effect of Rate of Loading upon the Static and Fatigue Strength of Plain Concrete in Compression," Magazine of Concrete Research, Vol. 25, No. 83. June, 1973, pp. 73-80.

58. Swamy, N., and Rigby, G., "Dynamic Properties of Hardened Paste, Mortar and Concrete," Materials and Structures, Paris, France, Vol. 4, No. 19, 1971, pp. 13-40.

59. Symonds, P. S., "Physical Plastic Behavior in Response of Structures to Dynamic Load," Behavior of Materials under Dynamic Loads, N. J. Huffington, Jr., Ed., American Society of Mechanical Engineers, New York, N.Y., 1965.

60. Takabayaski, M. T. “'Comparison of Dynamic Young's Modulus and Static Young's Modulus for Concrete," Réunion International des Laboratoires d'Etudes et d'Esn en Matériaux et Constructions, (RILEM), International Symposium on Non-Destructive Testing of Materials and Structures, Vol. 1, 1954, pp. 34-44.

61. Takeda, J., Tachikawa, H., and Fujimoto, K., "Mechanical Behavior of Concrete under High Rate Loading Than in Static Test," Symposium on Mechanical Behavior of Materials. Proceedings Society of Material Science, Kyoto, Japan, Vol. 2, 1974, pp. $479-486$.

62. Trott, J. J., and Fox, E. N., "Comparison of the Behavior of Concrete Beams under Static and Dynamic Loading." Magazine of Concrete Research, Vol. 11, No. 31, Mar., 1959, pp. 15-24.

63. Watstein, D., "Effect of Straining Rate on the Compressive Strength and Elastic Properties of Concrete," American Concrete Institute Journal, Vol. 49, No. 8, Apr., 1953, pp. 729-744.

64. Watstein, D., "Properties of Concrete at High Speed of Loading." STP No. 176. Symposium on Impact Testing. American Society of Mechanical Engineers, New York, N.Y., 1956, pp. 156-169. 